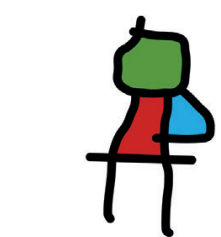

SEICAIP

\section{Allergologia et \\ immunopathologia}

Sociedad Española de Inmunología Clínica,

Alergología y Asma Pediátrica

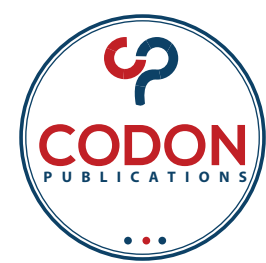

ORIGINAL RESEARCH

\title{
Pachymic acid inhibits inflammation and cell apoptosis in lipopolysaccharide (LPS)-induced rat model with pneumonia by regulating NF- $\kappa B$ and MAPK pathways
}

\author{
Yanjun Gui, Lijuan Sun, Rui Liu, Jinzhu Luo* \\ Department of Pediatrics, Dingxi People's Hospital, Dingxi, Gansu, China
}

Received 17 June 2021; Accepted 6 July 2021

Available online 1 September 2021

\section{KEYWORDS \\ apoptosis; \\ inflammation; \\ MAPK; \\ NF-kB; \\ pachymic acid (PA); \\ pneumonia}

\begin{abstract}
Pneumonia is a common infectious disease with high morbidity and mortality. It is caused by a variety of pathogenic microorganisms that infect the lung parenchyma. Anti-infective drugs are one of the preferred choices for the treatment of pneumonia. Pachymic acid (PA) is a lanolin triterpene compound from Poria cocos, which has antiemetic, anti-inflammatory, and anticancer properties. Although PA inhibits inflammatory response in a variety of diseases, its role in pneumonia is not clear. In this study, we established that PA improved histopathological changes in the lungs of rats with pneumonia. PA inhibited the expression of inflammatory cytokines in the serum of rats having pneumonia. In addition, PA inhibited the apoptosis of cells from rat lung tissues. Mechanically, PA inhibited inflammation and cell apoptosis via NF-KB and MAPK pathways. Therefore, PA could serve as a promising drug for treating pneumonia.

(c) 2021 Codon Publications. Published by Codon Publications.
\end{abstract}

*Corresponding author: Jinzhu Luo, Department of Pediatrics, Dingxi People’s Hospital, Dingxi, No. 22 Anding Road, Anding District, Dingxi City, Gansu Province 743000, China. Email address: l_jingz@163.com 


\section{Introduction}

Pneumonia is a common infectious disease, ${ }^{1}$ characterized by the lower respiratory tract infection by pathogens such as viruses, bacteria, and fungi. The morbidity and mortality rates because of pneumonia are increasing worldwide. Typical symptoms of pneumonia are chills, fever, pleurisy, cough, and purulent sputum. Pneumonia has become a leading cause of death in children aged less than 5 years, with about 1.3 million child deaths worldwide every year. ${ }^{2}$ A better understanding of the underlying mechanism of childhood pneumonia could contribute to the development of an effective and novel treatment to overcome this burden. ${ }^{3,4}$ Fighting infection is the primary need toward treating pneumonia. Since pneumonia is a disease caused by a variety of pathogenic microorganisms infecting the lung parenchyma, anti-infective drugs are the first choice for its treatment. ${ }^{5}$

Pachymic acid (PA) is a lanolin triterpene compound from Poria cocos. ${ }^{6}$ Studies have demonstrated that PA has antiemetic, anti-inflammatory, and anticancer properties. ${ }^{7}$ Previous investigations have shown that Poria could reduce inflammatory response to septic kidney and lung injuries. ${ }^{8}$ In particular, PA has an anti-inflammatory effect in the pulpitis model. ${ }^{9}$ Besides, PA protects cardiomyocytes from LPS-induced inflammation and apoptosis by regulating the extracellular signal-regulated kinase (ERK) and p38 mitogen-activated protein kinase (p38 MAPK) pathways. ${ }^{10}$ PA also competitively inhibits the biological activity of phospholipase A2 (PLA2) and reduces PLA2-mediated cell damage. ${ }^{6}$ In addition, PA inhibits the invasion of breast cancer by regulating nuclear factor kappa $B$ (NF-kB) pathway. ${ }^{11}$

NF-KB and mitogen-activated protein kinase (MAPK) signaling are important pathways of inflammation. Signaling from pathogens or host cells, such as pathogen-associated molecular patterns (PMAP), is recognized by various pattern recognition receptors and eventually triggers the activation of MAPK (p38, ERK, and c-JUN N-terminal kinase [JNK]) and NF-KB signaling pathways. ${ }^{12}$ These pathways activate gene transcription and promote the expressions of various cytokines and amplify the inflammatory response through positive feedback cascades. ${ }^{13}$ Several studies have provided evidence showing that NF-KB could serve as a target for treatment of pneumonia..$^{14}$

Although previous studies have shown that PA has anti-inflammatory effects and could inhibit the inflammatory response in a variety of diseases, its role in pneumonia is not clear. The purpose of this study was to elucidate the potential effects of PA on pneumonia and explore relevant regulatory mechanisms.

\section{Materials and methods}

\section{Animals and treatment}

The animal study was performed according to the National Institutes of Health Laboratory Animal Care and Use Guidelines. The study was approved by the Institutional Animal Care and Use Committee of the Dingxi People's Hospital. A total of 20 male rats weighing approximately 180-220 g were brought from Shanghai Slack Laboratory
Animal Co. Ltd. The rats were randomly divided into the following four groups ( $\mathrm{n}=5$ in each group): control group, Lipopolysaccharide (LPS) group (2 mg/kg LPS), LPS+PA group $(10 \mathrm{mg} / \mathrm{kg})$, and LPS+PA group $(20 \mathrm{mg} / \mathrm{kg})$. PA was purchased from BioCrick Biotech (Chengdu, Sichuan Province, China) and administered to rats intraperitoneally. To induce acute lung injury, rats were injected light anesthesia with $0.5 \%$ pentobarbital sodium intraperitoneally and intranasal administration of $2 \mathrm{mg} / \mathrm{kg}$ of LPS in sterile saline. After 3 days, the blood samples were collected and rats were sacrificed. Lungs were subjected to bronchoalveolar lavage fluid (BALF) collection.

\section{Hematoxylin and Eosin (HE) staining}

Freshly excised lung tissues were fixed with 10\% paraformaldehyde and embedded with paraffin and cut into $4-\mu \mathrm{m}$ slices. Then these sections were deparaffinized and subjected to $\mathrm{HE}$ (Sigma-Aldrich, Kenilworth, USA) staining. The total lung injury score was calculated as the sum of the scores of each variable (edema, neutrophilic infiltration, interstitial inflammation, and congestion). The scores ranged between 0 (normal) and 4 (severe), with the mean value recorded as a semi-quantitative histological indicator of lung injury.

\section{Lung wet-to-dry weight ratio measurement}

The lung samples were isolated after sacrifice of rats and weighed immediately. The samples were dried until the weight was stable. The wet-to-dry weight (W/D) ratio was obtained.

\section{Enzyme linked immunosorbent assay (ELISA)}

The lung tissues were lysed with phosphate buffer solution (PBS) and minced with magnetic beads. Then lysates were collected and centrifuged. Concentrations of TNF-a, Interleukin (IL)-1B, IL-6, and monocyte chemotactic protein (MCP) in lung tissues were measured by using the ELISA kit (R\&D Systems, Minneapolis, USA).

\section{Cell number in BALF}

The BALF samples were centrifuged at $1700 \mathrm{~g}$ for $10 \mathrm{~min}$ at $4^{\circ} \mathrm{C}$ and cell pellets were dissolved in saline. Cell numbers in the suspension were measured with an automatic blood cell counter (Sysmex E-25000; Toua-iyoudenshi Co. Ltd, Japan).

\section{TUNEL assay}

The slices were deparaffinized in xylene for $5 \mathrm{~min}$, hydrated, and rinsed in distilled water. Then the sections were incubated with $3 \% \mathrm{H}_{2} \mathrm{O}_{2}$ to block endogenous peroxidase activity and terminal deoxyribonucleotidyl transferase (TdT) reaction mixture for $1-2 \mathrm{~h}$ in a humidified chamber. Then reaction was stopped by rinsing sections 
in Stop Wash Buffer. The signals were detected after incubation with streptavidin-horseradish peroxidase (HRP) and diaminobenzidine (DAB) under light microscope.

\section{Immunoblot analysis}

Protein samples from tissues were separated through electrophoresis on sodium dodecylsulfate-polyacrylamide gel and transferred to polyvinylidene difluoride membranes. After blocking with $5 \%$ milk in TBST (20-mM tris-buffered saline, $500-\mathrm{mM} \mathrm{NaCl}, \mathrm{pH} 7.5$, and $0.1 \%$ Triton X-100) for $1 \mathrm{~h}$ at room temperature, the membranes were incubated with anti-Bax antibody (1:1000 dilution, ab32503, Abcam plc, Cambridge, UK), anti-GAPDH (1:5000 dilution, ab8245, Abcam plc), anti-B-cell lymphoma protein 2 (Bcl-2) (1:1000 dilution, ab32124, Abcam plc), anti-p65 (1:2000 dilution, ab32536, Abcam plc), anti-p-p65 (1:1000 dilution, ab76302, Abcam plc), anti-p38 (1:1000 dilution, ab170099, Abcam plc), anti-p-p38 (1:1000 dilution, ab178867, Abcam plc), antiERK (1:1000 dilution, ab184699, Abcam plc), and anti-p-ERK (1:1000 dilution, ab201015, Abcam plc) at $4^{\circ} \mathrm{C}$ overnight. Then membranes were washed for three times in TBST and conjugated with secondary antibody with horseradish peroxidase (Sigma, Kenilworth, USA) at room temperature for $1 \mathrm{~h}$. The membranes were analyzed with enhanced chemiluminescence $(E C L)$ reagent (Sigma, Kenilworth, USA).

\section{Statistics}

GraphPad 5.0 software was used for statistical analysis. Data were represented as mean \pm SD. Student's $t$-test was used for comparisons, and $\mathrm{P}<0.05$ was considered as significant.

\section{Results}

\section{PA alleviates Lipopolysaccharide (LPS)-induced lung injury in Rats}

In order to explore the therapeutic effects of PA in LPSinduced lung injury in rats, four groups of rats were pre-treated with or without PA. After induction of LPS in rats, histological changes in each group were analyzed through HE staining. LPS treatment induced lung injury, such as alveolar exudate, edema, and inflammatory cell infiltration, in rats compared with the control group (Figure 1A). However, PA treatment significantly alleviated these phenomena in a dose-dependent manner (Figure 1A). Consistently, PA treatment reversed the elevated W/D ratio of lung tissue induced by LPS (Figure 1B). Taken together, these results indicated that PA could attenuate lung injury induced by LPS.

\section{$P A$ relieves inflammation induced by LPS in rats}

Induction of LPS was accompanied with elevated level of TNF- $\alpha, I L-6, I L-1 B$, and MCP-1. To delineate the role of PA in LPS-induced inflammatory cytokine production, the respective concentrations of TNF- $\alpha$, IL-6, MCP-1, and IL-1B were detected in the lung tissues of rats. As shown in Figure 2, LPS stimulation significantly elevated the levels of TNF- $a$, IL-6, MCP-1, and IL-1B in lung tissues. However, administration of PA lowered TNF-a level to half of that in the LPS group, and the levels of IL-6, MCP-1, and IL- $1 B$ in the PA group were decreased to less than $50 \%$ that in the LPS group. These results suggested that PA treatment could relieve LPS-induced inflammation in mice.

\section{$P A$ relieves cell numbers in BALF of rats induced by LPS}

In order to analyze cell numbers in BALF, the total cell number, neutrophil number, and white cell number were measured. Clear elevation in the total cell number, neutrophil number, and white cell number in the BALF of LPS-induced rats were observed. After PA treatment, the total cell number, neutrophil number, and white cell number were decreased in a dose-dependent manner (Figures 3A-3C). Taken together, the PA treatment effectively reduced the total cell number, neutrophil number, and white cell number in the BALF of LPS-induced rats.

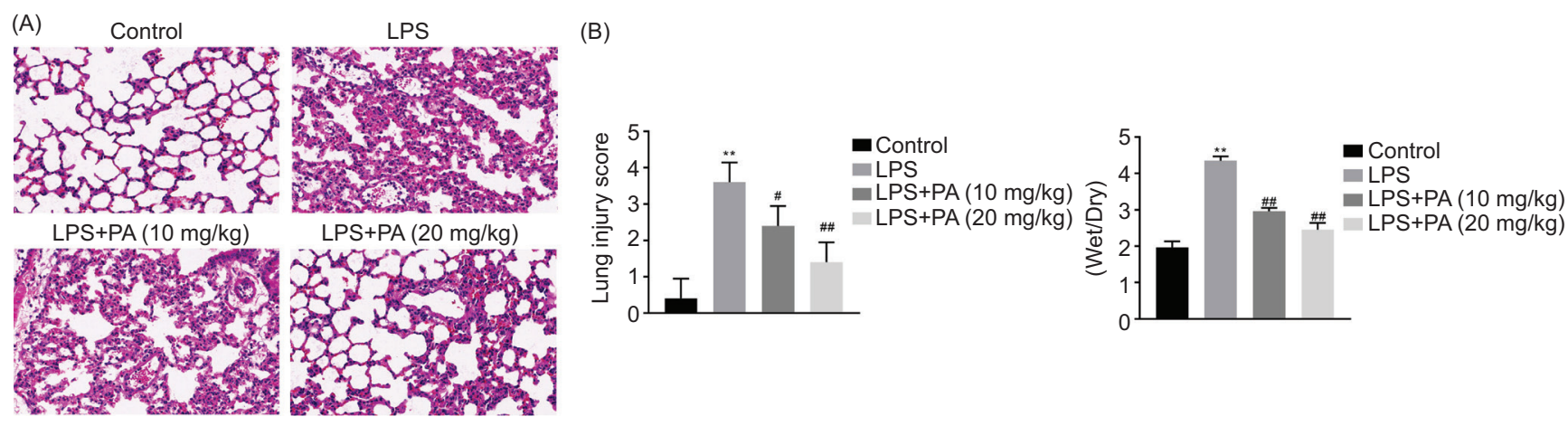

Figure 1 PA alleviates LPS-induced lung injury in Rats. (A) The histological changes of lung tissue in control, LPS, LPS+10 mg/ $\mathrm{kg}$ PA, and LPS $+20 \mathrm{~g} / \mathrm{kg}$ PA groups. (B) The wet/dry weight (W/D) ratio of the lung tissues in control, LPS, LPS+10 mg/kg PA, and LPS+20 g/kg PA groups. ${ }^{* *} \mathrm{P}<0.01$ versus the control group; ${ }^{\# P}<0.05,{ }^{\# \#}<0.01$ versus the LPS group. 

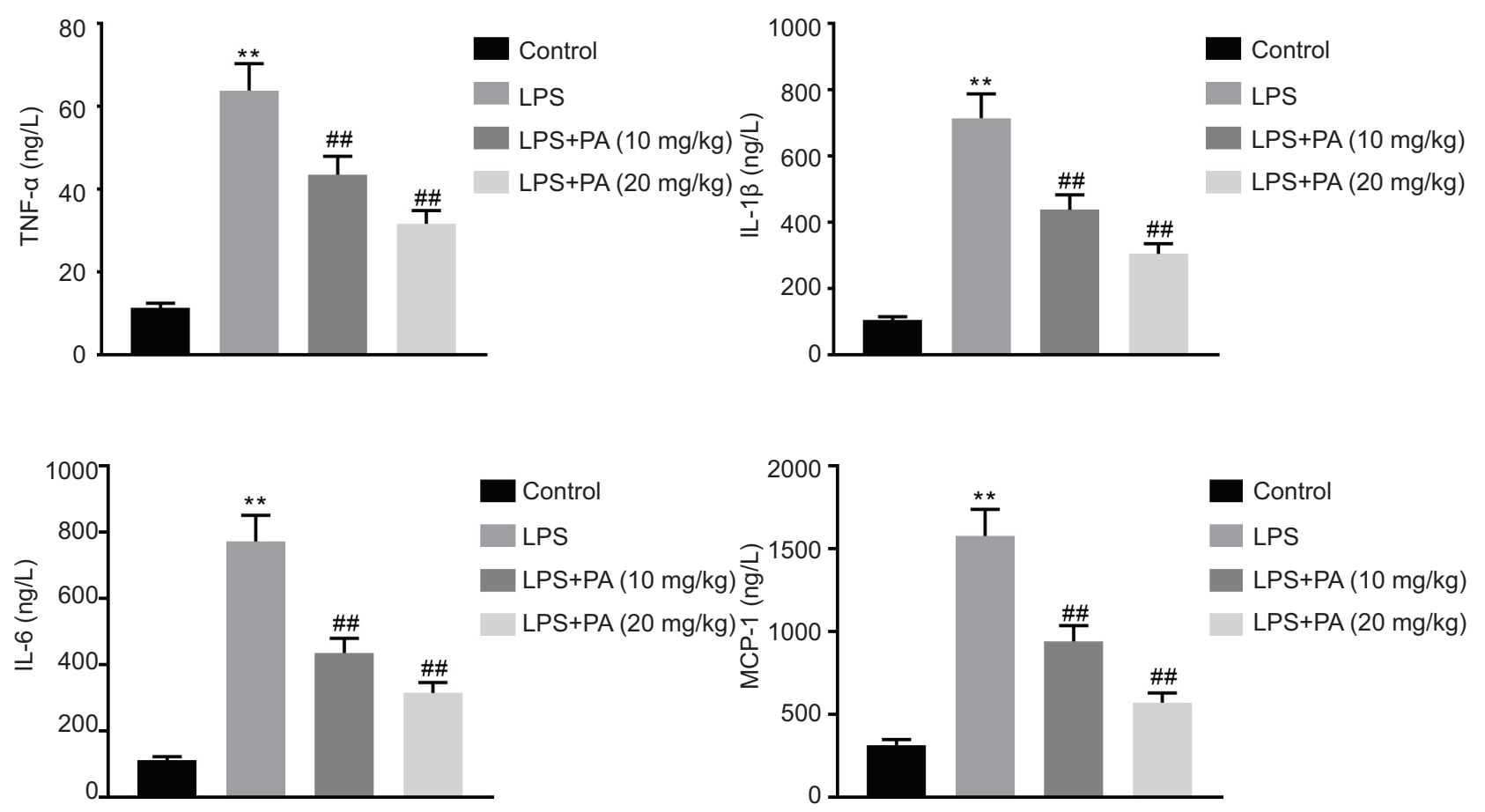

Figure 2 PA relieves inflammation induced by LPS in rats. IL-6, IL-1B, TNF-a, and MCP-1 level in control, LPS, LPS+10 mg/kg PA, and LPS $+20 \mathrm{~g} / \mathrm{kg}$ PA groups. **P $<0.01$ versus the control group; ${ }^{\#} \mathrm{P}<0.01$ versus the LPS group.
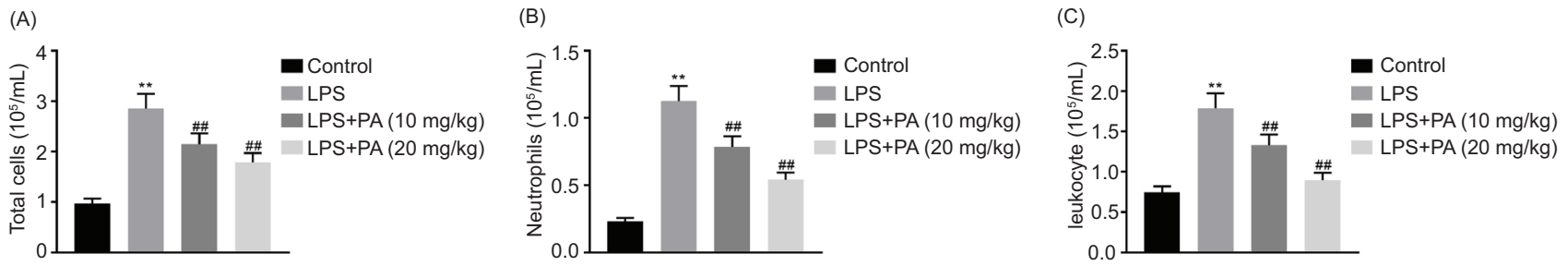

Figure 3 PA relieves cell number in the BALF of LPS-induced rats. (A-C) The total cell number, neutrophil, and white cell number in control, LPS, LPS $+10 \mathrm{mg} / \mathrm{kg}$ PA, and LPS $+20 \mathrm{~g} / \mathrm{kg}$ PA groups. ${ }^{* *} \mathrm{P}<0.01$ versus the control group; \#\# $<0.01$ versus the LPS group.

\section{PA inhibits cell apoptosis in rats induced by LPS.}

The cell apoptosis in lung tissues was detected by TdTmediated dUTP Nick-End Labeling (TUNEL) assay. As expected, LPS induction in rats led to increased number of apoptotic cells in rats, which was evidenced by increased number of TUNEL positive cells (Figure 4A). PA treatment significantly reduced the number of TUNEL positive cells (Figure 4A). Also, the expression levels of $\mathrm{Bax}$ and $\mathrm{Bcl}-2$ were detected in lung tissues. LPS treatment enhanced the Bax level and reduced Bcl-2 level. However, alterations in the levels of $\mathrm{Bax}$ and $\mathrm{Bcl}-2$ were reversed by PA treatment (Figure 4B). Thus these results suggested that PA could inhibit cell apoptosis in rats induced by LPS.

\section{$P A$ represses NF-KB and MAPK signaling pathways}

NK-kB and MAPK signaling pathways were involved in LPSinduced inflammation model. The possible association between PA and these signaling pathways was analyzed.
LPS treatment elevated the levels of p-p65, p-p38, and $\mathrm{p}-\mathrm{ERK} 1 / 2$ in lung tissues of rats as compared to that from the control group. However, PA challenge significantly reduced the expression levels of $p-p 65, p-p 38$, and $\mathrm{p}$-ERK1/2 in the lung tissues of LPS-induced lung injury model (Figure 5). Thus, these findings suggested that PA could suppress NF-KB and MAPK signaling pathways.

\section{Discussion}

Pneumonia is a major disease threatening human life. Inflammatory response and apoptosis of lung epithelial cells have important effects on the development and prognosis of pneumonia. ${ }^{15,16}$ Overexpression of cytokines is also an important cause of pneumonia; hence, regulation of body's inflammatory response is an effective means to treat pneumonia. ${ }^{17}$ Based on the importance of apoptosis and inflammatory response in the progression of pneumonia, many studies have focused on investigating targets related to apoptosis and inflammatory response so as to 
(A)

Control

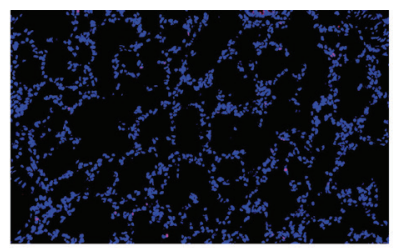

(B)
LPS

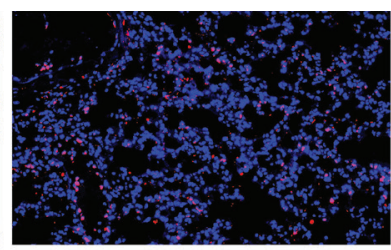

20
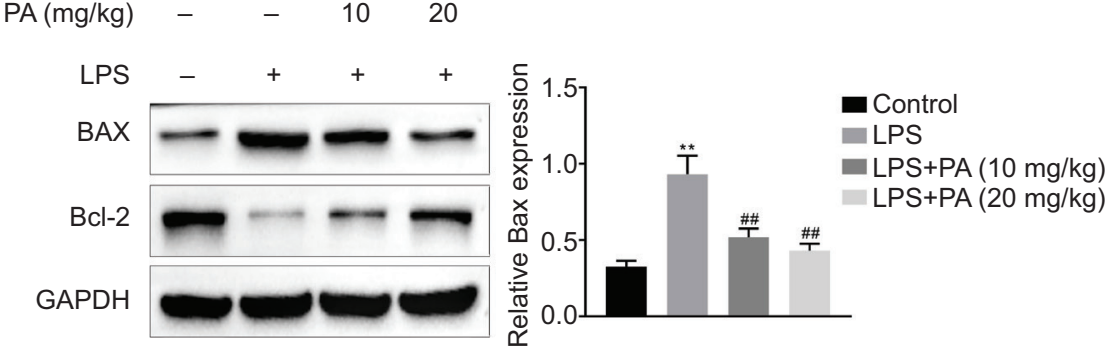

LPS+PA (10 mg/kg)

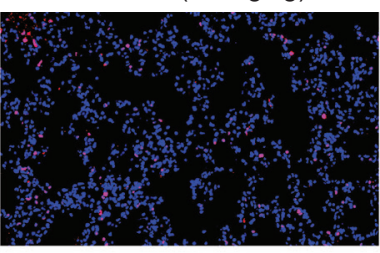

LPS+PA (20 mg/kg)

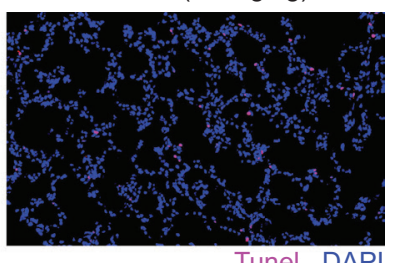

Tunel DAPI

Figure 4 PA inhibits cell apoptosis in rats induced by LPS. (A) TUNEL staining of lung tissues in control, LPS, LPS+10 mg/kg PA, and LPS $+20 \mathrm{~g} / \mathrm{kg}$ PA groups. (B) Immunoblot assay detected the protein levels of Bax and Bcl-2 in control, LPS, LPS+10 mg/kg PA, and LPS $+20 \mathrm{~g} / \mathrm{kg}$ PA groups. The relative expression related to the GAPDH was used as 1 for comparison. ${ }^{* *} \mathrm{P}<0.01$ versus the control group; \#P $<0.01$ versus the LPS group.
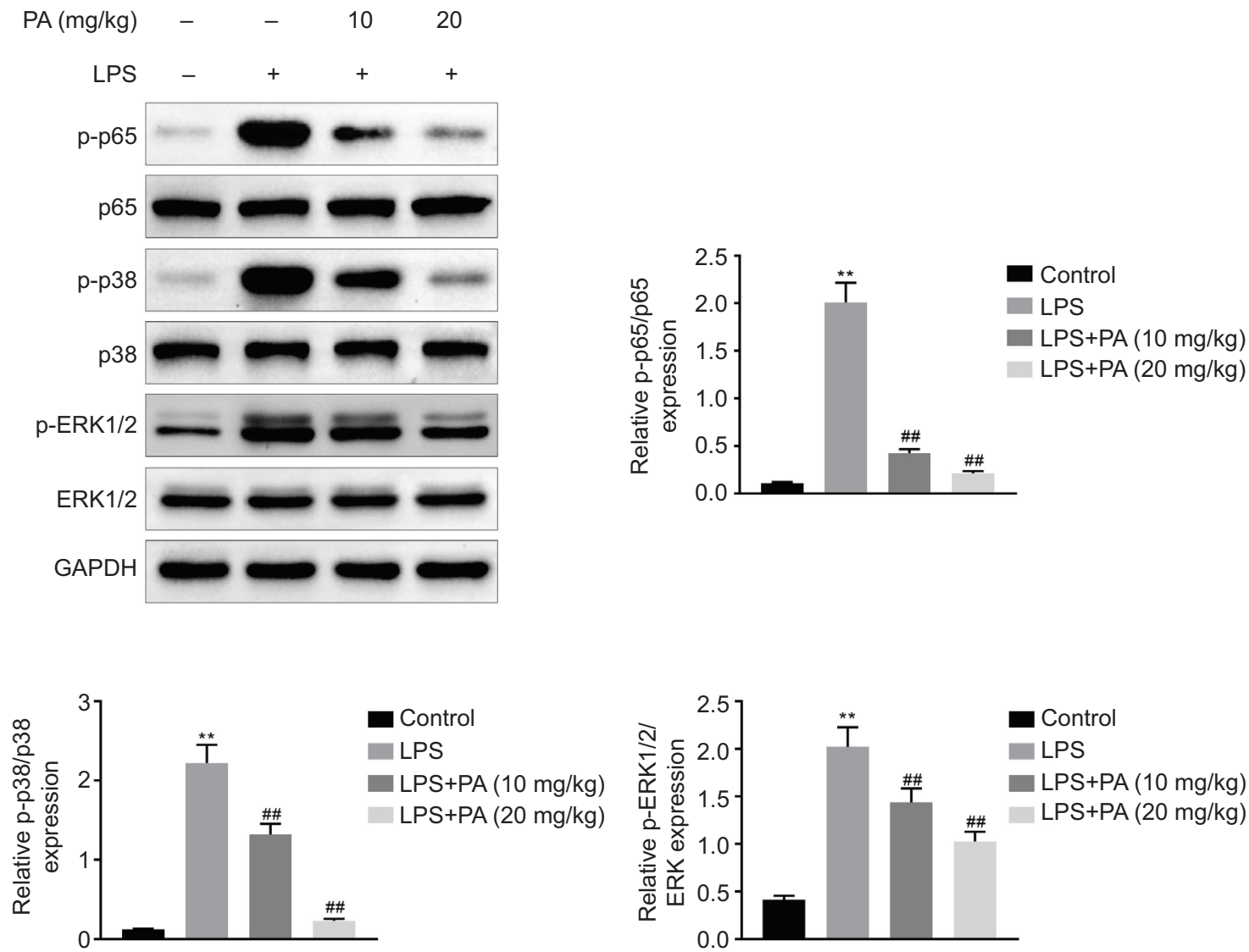

Figure 5 PA represses NF- $\mathrm{kB}$ and MAPK signaling pathways. Immunoblot assay detected the protein levels of p-p65, p65, p-p38, p38, p-ERK1/2, and ERK1/2 in control, LPS, LPS $+10 \mathrm{mg} / \mathrm{kg}$ PA, and LPS $+20 \mathrm{~g} / \mathrm{kg}$ PA groups. The relative expression related to the GAPDH was used as 1 for comparison. ${ }^{* *} P<0.01$ versus the control group; ${ }^{\#} P<0.01$ versus the LPS group. 
improve the prognosis of patients and improve their quality of life..$^{18}$ Importantly, PA, a lanolin triterpene compound, was identified as a potential factor for the treatment of pneumonia. However, the precise mechanism needs further investigation.

The effect of PA on the progression of pneumonia in vitro was determined by HE, ELISA, TUNEL, and Immunoblot assays. The multiple biological activities of PA have been widely revealed in a variety of studies.,11 PA suppressed TGF-B1-induced renal fibrosis and proliferation via MAPK pathway. ${ }^{19}$ Similarly, the effect of PA on this pathway in lung tissue cells was also observed. Also, PA could activate AMP-activated protein kinase (AMPK) pathway to attenuate fibroblast activation and defects of extracellular matrix remodeling in renal fibrosis. ${ }^{11}$ It could also protect against cerebral ischemia injury by targeting PI3K/Akt pathway. ${ }^{10}$ Also, PA enhanced melatonin by inhibition of AKI-to-CKD transition through mediating growth arrest-specific 6 and nuclear factor-erythroid-2-related factor 2 (Gas6/Nrf2) axis. ${ }^{10}$ Another study showed that PA impaired glucose metabolism and stimulated mitochondrial apoptosis. ${ }^{8}$ In the present study, the alterations of Bax and Bcl-2 were reversed by PA treatment, suggesting that PA suppressed cell apoptosis in rats with lung injury. On the contrary, PA plays its roles in cancer treatment due tobecause of its pro-apoptotic activity. This different result could be due to different diseases. Besides, PA challenge significantly reduced the expression levels of $p-p 65$, $\mathrm{p}-\mathrm{p} 38$, and $\mathrm{p}-\mathrm{ERK} 1 / 2$ in the lung tissues of LPS-induced lung injury model. These studies provided the evidence that PA affected multiple cellular processes via multiple mechanisms.

The effect of NF-KB/MAPK pathway on the progression of pneumonia have been clearly revealed. ${ }^{20-22}$ NF-KB pathway could serve as a potential target for the treatment of pneumonia. Several drugs have been used for treating pneumonia through targeting NF-kB pathway. ${ }^{23}$ For example, knockdown of circ-UQCRC2 ameliorated LPS-induced injury in lung MRC-5 cells by NF-kB pathway. ${ }^{24}$ Another study has indicated that miR-143-3p affected pulmonary inflammatory factors and cell apoptosis in mice with mycoplasmal pneumonia through NF-kB pathway. ${ }^{13}$ Notably, MAPK pathway also had critical effects on progression of pneumonia. ${ }^{23}$ A study has indicated that cardiolipin-mediated PPARy S112 phosphorylation impaired IL-10 production and inflammation resolution via MAPK pathway during bacterial pneumonia. The nucleotide-binding oligomerization domain (NOD)-like receptors could mediate inflammatory lung injury during plateau hypoxia exposure via MAPK pathway, suggesting the effects on pneumonia. ${ }^{23,25}$ Although several studies have confirmed the effects of PA on the phenotypes of inflammation and apoptosis via NF-KB and MAPK pathways, this is the first evidence showing that PA affected and interfered with lung injury through this signaling pathway. To some extent, this study explained the effect of PA on the apoptosis and inflammation in lung injury in model rats. Our study innovatively found that PA could be used as a potential therapeutic agent for lung injury, and its precise mechanism of action is still worthy of further study. These studies, together with our findings, confirmed that both NF-KB and MAPK pathways could serve as promising therapeutic targets for the treatment of pneumonia.
In summary, the present study demonstrated that PA improved the histopathological alterations in lung tissues in model rats with pneumonia. PA inhibited the expression levels of inflammatory cytokines in rat serum and suppressed cell apoptosis from rat lung tissue. Mechanically, PA inhibited inflammation and cell apoptosis via NF- $k B$ and MAPK pathways. Therefore, PA could serve as a promising drug for the treatment of pneumonia.

\section{Competing interests}

The authors state that there are no conflicts of interest to disclose.

\section{Ethics approval}

Ethical approval was obtained from the Ethics Committee of Dingxi People's Hospital.

\section{Contribution of authors}

Yanjun Gui and Lijuan Sun designed the study and supervised data collection. Rui Liu analyzed and interpreted the data. Jinzhu Luo reviewed the draft of manuscript and prepared it for publication. All authors have read and approved the final manuscript.

\section{References}

1. Hur I, Ozkan S, Halici A, Abatay K, Usul E, Cetin E, et al. Role of plasma presepsin, procalcitonin and $\mathrm{C}$-reactive protein levels in determining the severity and mortality of communityacquired pneumonia in the emergency department. Signa Vitae. 2020;1:8. https://doi.org/10.22514/sv.2020.16.0034

2. Schnickel GT, Greenstein S, Berumen JA, Elias N, Sudan DL, Conzen KD, et al. Understanding the impact of pneumonia and other complications in elderly liver transplant recipients: An analysis of NSQIP transplant. Transplant Direct. 2021;7(5):e692. https://doi.org/10.1097/TXD.0000000000001151

3. Kiboshi T, Kotani T, Konma J, Makino H, Matsuda S, Suzuka T, et al. Comparison of therapeutic effects of combination therapy with prednisolone and tacrolimus or azathioprine on progressive interstitial pneumonia with systemic sclerosis. Modern Rheumatol. 2021:1-17. https://doi.org/10.1080/143975 95.2021.1918864

4. Guo JG, Kong Q, Liu C, Kang TS, Qin C. Evaluating the Health Risks of pneumonia from airborne bacterial communities using $16 \mathrm{~S}$ rDNA sequences of pneumonia-related pathogens. Biomed Environ Sci (BES). 2021;34(4):265-71. https://doi.org/10.3967/ bes2021.035

5. Gao HM, Ambroggio L, Shah SS, Ruddy RM, Florin TA. Predictive value of clinician "Gestalt" in pediatric community-acquired pneumonia. Pediatrics. 2021; 147(5) e2020041582. https://doi.org/10.1542/peds.2020-041582

6. Cai H, Cheng $\mathrm{Y}$, Zhu Q, Kong D, Chen X, Tamai I, et al. Identification of triterpene acids in poria cocos extract as bile acid uptake transporter inhibitors. Drug metabolism and disposition. 2021;49(5):353-60. https://doi.org/10.1124/ dmd.120.000308

7. Akihisa T, Uchiyama E, Kikuchi T, Tokuda H, Suzuki T, Kimura Y. Anti-tumor-promoting effects of 25-methoxyporicoic acid A 
and other triterpene acids from Poria cocos. J Natural Prod. 2009;72(10):1786-92. https://doi.org/10.1021/np9003239

8. Akihisa T, Mizushina Y, Ukiya M, Oshikubo M, Kondo S, Kimura Y, et al. Dehydrotrametenonic acid and dehydroeburiconic acid from Poria cocos and their inhibitory effects on eukaryotic DNA polymerase alpha and beta. Biosci, Biotechnol Biochem. 2004;68(2):448-50. https://doi.org/10.1271/bbb.68.448

9. Yasukawa K, Kaminaga T, Kitanaka S, Tai T, Nunoura Y, Natori S, et al. 3 beta-p-hydroxybenzoyldehydrotumulosic acid from Poria cocos, and its anti-inflammatory effect. Phytochemistry. 1998;48(8):1357-60. https://doi.org/10.1016/ S0031-9422(97)01063-7

10. Xu H, Wang Y, Jurutka PW, Wu S, Chen Y, Cao C, et al. 16Alpha-hydroxytrametenolic acid from poria cocos improves intestinal barrier function through the glucocorticoid receptor-mediated PI3K/Akt/NF-kappa B pathway. J Agr Food Chem. 2019;67(39):10871-9. https://doi.org/10.1021/acs.jafc.9b04613

11. Gapter L, Wang Z, Glinski J, Ng KY. Induction of apoptosis in prostate cancer cells by pachymic acid from Poria cocos. Biochem Biophy Res Comm. 2005;332(4):1153-61. https://doi. org $/ 10.1016 /$ j.bbrc.2005.05.044

12. Yang J, Deng F, Pan N. [Butein promotes the role of $\mathrm{mH} 2 \mathrm{~A}$ in MAPK signaling pathway through targeting GRP78 in regulating biological behaviors of melanoma]. Zhong Nan Da Xue Xue Bao Yi Xue Ban. (J Central South Univ Med Sci. 2017;42(10):112935. https://doi.org/10.11817/j.issn.1672-7347.2017.10.001

13. Wang Z, Ka SO, Lee Y, Park BH, Bae EJ. Butein induction of $\mathrm{HO}-1$ by $\mathrm{p} 38 \mathrm{MAPK} / \mathrm{Nrf2}$ pathway in adipocytes attenuates high-fat diet induced adipose hypertrophy in mice. Eur J Pharmacol. 2017;799:201-10. https://doi.org/10.1016/j. ejphar.2017.02.021

14. Swiderek E, Kalas W, Wysokinska E, Pawlak A, Rak J, Strzadala L. The interplay between epigenetic silencing, oncogenic KRas and HIF-1 regulatory pathways in control of BNIP3 expression in human colorectal cancer cells. Biochem Biophy Res Comm. 2013;441(4):707-12. https://doi.org/10.1016/j. bbrc.2013.10.098

15. El Gharib K, Masri K, Daoud Z, Sfeir T. Community-acquired Acinetobacter calcoaceticus pneumonia in a patient with a gammaglobulinaemia. New Microbes New Infect. 2021;41:100870. https://doi.org/10.1016/j.nmni.2021.100870

16. Sultana M, Alam NH, Ali N, Faruque ASG, Fuchs GJ, Gyr N, et al. Household economic burden of childhood severe pneumonia in Bangladesh: A cost-of-illness study. Arch Dis Child. 2021; 320834. https://doi.org/10.1136/archdischild-2020-320834
17. Raftery NB, Murphy CF, Donohoe CL, O’Connell B, King S, Ravi N, et al. The complexity of defining postoperative pneumonia following esophageal cancer surgery: A spectrum of lung injury rather than a simple infective complication? Ann Surg. 2021. https://doi.org/10.1097/SLA.0000000000004873

18. Nair R, Gao Y, Vaughan-Sarrazin MS, Perencevich E, Girotra S, Pandey A. Risk-standardized home time as a novel hospital performance metric for pneumonia hospitalization among medicare beneficiaries: A retrospective cohort study. J Gen Intern Med. 2021. https://doi.org/10.1007/s11606-021-06712-w

19. Song $Z$, Bi K, Luo X, Chan K. The isolation, identification and determination of dehydrotumulosic acid in Poria cocos. Anal Sci Int J Jap Soc Anal Chem. 2002;18(5):529-31. https://doi. org/10.2116/analsci.18.529

20. Wang SC, Dowhan DH, Eriksson NA, Muscat GE. CARM1/PRMT4 is necessary for the glycogen gene expression programme in skeletal muscle cells. Biochem J. 2012;444(2):323-31. https:// doi.org/10.1042/BJ20112033

21. Jiang $T$, Xia Y, Lv J, Li B, Li Y, Wang S, et al. A novel protein encoded by circMAPK1 inhibits progression of gastric cancer by suppressing activation of MAPK signaling. Mol Cancer. 2021;20(1):66. https://doi.org/10.1186/s12943-021-01358-y

22. Zhang BF, Jiang $H$, Chen J, Guo X, Hu Q, Yang S. KDM3A inhibition attenuates high concentration insulininduced vascular smooth muscle cell injury by suppressing MAPK/NFkappaB pathways. Int J Mol Med. 2018;41(3):1265-74. https://doi. org/10.3892/ijmm.2017.3351

23. Xia L, Xiao X, Liu WL, Song Y, Liu TJJ, Li YJ, et al. Coactosinlike protein CLP/Cotl1 suppresses breast cancer growth through activation of IL-24/PERP and inhibition of non-canonical TGFbeta signaling. Oncogene. 2018;37(3):323-31. https:// doi.org/10.1038/onc.2017.342

24. Szuster-Ciesielska A, Mizerska-Dudka M, Daniluk J, KandeferSzerszen $M$. Butein inhibits ethanol-induced activation of liver stellate cells through TGF-beta, NFkappaB, p38, and JNK signaling pathways and inhibition of oxidative stress. J Gastroenterol. 2013;48(2):222-37. https://doi.org/10.1007/ s00535-012-0619-7

25. Hu QP, Huang XY, Peng F, Yang H, Wu C. MS275 reduces seizure-induced brain damage in developing rats by regulating p38 MAPK signaling pathways and epigenetic modification. Brain Res. 2020;1745:146932. https://doi.org/10.1016/j. brainres.2020.146932 